\title{
Research Advances in Ultrasound Imaging for Tumor in Situ
}

Ping Wang, MD ${ }^{a}$, Lihong Sun, PhD ${ }^{a}$, Suhui Sun, MD ${ }^{a}$, Menghong Xu, MD ${ }^{a}$, Lulu Zhang, MD ${ }^{a}$, Jinxia Zhang, MD ${ }^{a}$, Liquan Gao, PhD ${ }^{a}$, Qingfeng Chen, PhD ${ }^{b}$, Xiaolong Liang, PhD ${ }^{a, *}$

${ }^{a}$ Department of Ultrasound, Peking University Third Hospital, Beijing, China; ${ }^{b}$ Institute of Molecular and Cell Biology, Agency for Science, Technology and Research, Singapore, Singapore

\begin{abstract}
Known for being highly sensitive and noninvasive, ultrasound imaging using microbubble contrast agents is widely used in the clinic. To use ultrasound to image tissue beyond the vasculature, researchers have developed strategies that include nanobubbles, ultrasound contrast agents generated in situ, and gene expression of ultrasound contrast agents in situ. All of these strategies offer the capability of targeting tumor cells, intratumoral imaging tumor cells and require just a small incision or no incision. In this review, we will first describe the application of nanobubbles acting as ultrasound contrast agents. Then, we will briefly introduce the stimuli-responsive formulations to generate ultrasound contrast agents in situ. Finally, we will provide an overview of the use of state-of-the-art of gene expression of ultrasound contrast agents in situ to monitor cellular location and function inside living organisms.
\end{abstract}

Key words: Ultrasound contrast agents; Nanobubbles; Gene expression; Ultrasound

Advanced Ultrasound in Diagnosis and Therapy 2020;04:169-175

DOI: 10.37015/AUDT.2020.190040

\section{Introduction}

"A cloud of echoes between the undulating margins of the aortic root" was observed after injection of agitated saline in 1968, proving for the first time that bubbles in the agitated saline could be used as ultrasound contrast agent for echocardiography [1]. The United States approved the first human ultrasound contrast agent, Albunex, in 1994 [2]. Since then, ultrasound contrast agents have been used to improve the sensitivity and accuracy of conventional ultrasound imaging [3]. All commercially available ultrasound contrast agents are currently microbubbles (MBs), which are typically designed with a diameter of $1-8 \mu \mathrm{m}$ to serve as blood pool agents $[4,5]$. These micro-sized bubbles are, however, too large to pass through the vasculature[6]. To penetrate deep into tissue, visualize cellular functions, and load therapeutic agents, tumor in situ ultrasound imaging is necessary [7].

Tumor in situ ultrasound imaging is a new strategy for visualizing cells or structures by using a new generation contrast agent. Among the promising solutions used for this imaging approach are nanobubbles (NBs) [8,9], ultrasound contrast agents generated in situ [10], and gene expression of ultrasound contrast agents in situ $[11,12]$. These new generation contrast agents exhibit several advantages over MBs: (1) imaging tumor cells beyond the vasculature; (2) targeting tumor by the enhanced permeability and retention (EPR) effect or by conjugating targeted molecules on the surface; (3) stimuli-responsive to generate ultrasound contrast agents in situ; and (4) gene expression of ultrasound contrast agents in situ to directly monitor tumor location.

Advanced nanotechnology has enabled the development of many new types of MBs for ultrasound imaging, but the exploitation of tumor in situ ultrasound imaging is still in its infancy (Table 1). We believe that with the rise of sophisticated advancements in nanomedicine, in situ ultrasound imaging agents would receive more attention in future imaging research and clinic practice. This review aims to provide an overview of the state-of-the-art of the tumor in situ ultrasound

\footnotetext{
* Corresponding author: Department of Ultrasound, Peking University Third Hospital, Beijing 100191, China

e-mail:xiaolong_liang@bjmu.edu.cn
} 
imaging strategies.

\section{Contrast Nanobubbles}

NBs acting as ultrasound contrast agents have increasingly received attention in molecular ultrasound imaging for tumors in situ. NBs usually exhibit a uniform size of $380-780 \mathrm{~nm}$, which is compatible with the pore sizes of leaky tumor blood vessels [16]. Different from MBs, NBs are able to passively leak through tumor blood vessels by EPR effect [17].

Table 1 Tumor in situ ultrasound imaging strategies

\begin{tabular}{|c|c|c|c|c|}
\hline Contrasts & Classification & Advantages & Disadvantages & Ref. \\
\hline \multirow[t]{4}{*}{ Nanobubbles } & Imaging & Passing through vasculature & Poor echogenic ability & {$[4,17]$} \\
\hline & Loading drugs & Imaging and therapy & Poor drug loading ability & {$[13]$} \\
\hline & Targeting & $\begin{array}{l}\text { Accumulating more in tumor } \\
\text { tissue }\end{array}$ & $\begin{array}{l}\text { Some tumor cells may negatively express } \\
\text { receptor ligand }\end{array}$ & {$[14,19]$} \\
\hline & Multifunction & Imaging and treating tumor & $\begin{array}{l}\text { Difficult to fabricate multifunctional } \\
\text { nanobubbles }\end{array}$ & [20] \\
\hline \multirow[t]{4}{*}{$\begin{array}{l}\text { Ultrasound contrast agents } \\
\text { generated in situ }\end{array}$} & Heat responsive & $\begin{array}{l}\text { Treating tumor and release } \\
\text { contrast agents }\end{array}$ & Harmful to normal tissue & [27] \\
\hline & $\mathrm{pH}$ responsive & Noninvasive & $\begin{array}{l}\mathrm{pH} \text { value may not low enough to trigger the } \\
\text { generation of contrast agents in some tumors }\end{array}$ & [26] \\
\hline & $\begin{array}{l}\text { Near-infrared (NIR)/ } \\
\text { Ultraviolet responsive }\end{array}$ & Noninvasive & Short tissue penetrating depth & [15] \\
\hline & Enzymatic responsive & Noninvasive & Enzyme specifically dependent & [28] \\
\hline $\begin{array}{l}\text { Gene expression of ultrasound } \\
\text { contrast agents in situ }\end{array}$ & Gas vesicles & Imaging living organism & $\begin{array}{l}\text { The production process of genetic encoding } \\
\text { gas vesicles is complex }\end{array}$ & [30,31] \\
\hline
\end{tabular}

At present, researchers are searching the potential of NBs for contrast enhancement, loading drugs, and tumortargeting. For example, Yin and coworkers fabricated a lipid NBs, constructing them with phospholipid shells to enclose the $\mathrm{C}_{3} \mathrm{~F}_{8}$ gas core, for enhanced ultrasound imaging of tumors [18]. The constructed NBs, with uniform nano-size $436.8 \pm 5.7 \mathrm{~nm}$, were found to provide ultrasonically similar contrast enhancement to MBs in in vitro study. In in vivo tumor imaging study, researchers found that the MBs rarely showed contrast enhancement after 15 minutes, while the NBs offered contrast enhancement lasting more than 15 minutes, with this persistence perhaps due to the accumulation of NBs in the tumor site (Fig.1).

Although NBs can accumulate in tumor passively by the EPR effect, they appear to have poor tumor selectivity. To make NBs more selective, they can be modified with targeting molecules that improve their anti-tumor efficacy. Targeting molecules may include RGD (Arg-Gly-Asp) peptide and antibody. Yang et al. investigated the possibility of increasing the accumulation of affibody-conjugated NBs in breast cancer BT-474 site [19]. They observed a large number of affibody-conjugated NBs on the surface of BT-474 cells by inverted fluorescence microscopy, and the in vitro contrast study proved that the affibody-conjugated NBs exhibited ultrasonic contrast enhancement that was similar to SonoVue MBs. In in vivo study, the affibody-conjugated NBs had better ultrasonic contrast enhancement than the SonoVue MBs and unconjugated NBs.

Scientists are interested in developing NBs not only for the purpose of ultrasound contrast imaging, but also for the purpose of loading drugs to improve tumor treatment. Zhang et al. constructed multifunctional targeted poly (lactic-co-glycolic acid) (PLGA) NBs (denoted $\mathrm{mAb} \mathrm{HLA}_{\mathrm{HL}} / \mathrm{MTX} / \mathrm{PLGA}$ ) [20]. By filling them with perfluorocarbon gas, the multifunctional nanobubbles served as ultrasound contrast agent for tumor imaging and enhanced high intensity focused ultrasound (HIFU) ablation efficiency. Loaded with methotrexate, the multifunctional NBs can serve as nanocarriers for treating tumors. Tumor targeting ability can also be improved by conjugating the multifunctional NBs with monoclonal anti-HLA-G antibodies. Both in vitro and in vivo study have demonstrated that the multifunctional NBs have excellent ultrasound contrast ability, tumor-targeted accumulation, and drug-loading and non-invasive HIFU therapy potential (Fig. 2). 

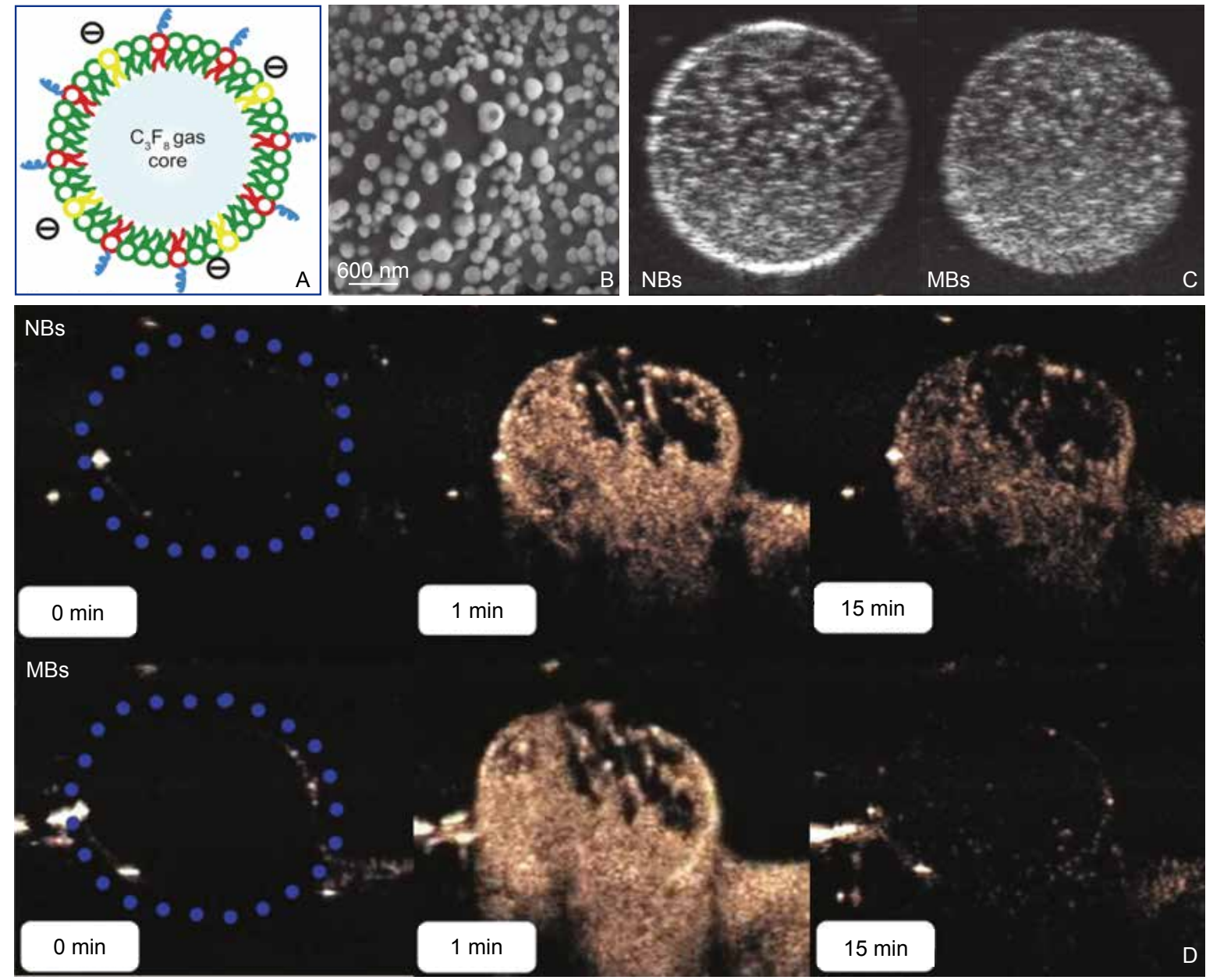

Figure 1 The NBs for ultrasound imaging. (A) Structure of the NBs; (B) Surface morphology of the NBs as visualized using scanning electron microscopy; (C) Representative in vitro ultrasonic images of NBs and MBs at concentration of $8.0 \times 10^{5}$ bubbles/ml, which shows that NBs exhibit better ultrasound contrast ability than MBs; (D) Representative subcutaneous tumor images before (blue dotted line) and after the injection of NBs compared with MBs at various time points $(0,1$, and 15 minutes). The NBs exhibit longer contrast-enhanced time than MBs. (Reproduced with permission from [18])

\section{Ultrasound Contrast Agents Generated in Situ}

Clinically available ultrasound contrast gasfilled MBs have undesirable intrinsic drawbacks that include short half-life and low stability in blood [21]. Researchers are actively working to prolong the half-life and improve the stability of MBs for in vivo ultrasound imaging [22-25]. Researchers are also exploring stimuliresponsive formulations to generate ultrasound contrast agents in situ ( $\mathrm{pH}$, heat, enzyme, light). Min et al. reported a $\mathrm{pH}$-controlled $\mathrm{CO}_{2}$-generating theranostic nanoparticle system consisting of doxorubicin-loaded $\mathrm{CaCO}_{3}$ for ultrasound imaging and treating tumors [26]. The acidic tumor micro-environment can hydrolyze $\mathrm{CaCO}_{3}$ to generate $\mathrm{CO}_{2}$ bubbles, which could trigger the release of doxorubicin and act as ultrasound contrast agents for tumor imaging. Ex vivo research showed that the amount of $\mathrm{CO}_{2}$ generated at $\mathrm{pH}=6.8$ is 1.59 times higher than the amount of $\mathrm{CO}_{2}$ generated at $\mathrm{pH}=7.4$.
And the nanoparticle system produced significantly better ultrasound contrast images at $\mathrm{pH}=6.8$. An intratumoral injection method was used to demonstrate the potential of the nanoparticle system for ultrasound imaging of the tumor site. Thirty minutes after injection, enhancement in ultrasound images was observed, and it lasted up to 120 minutes. The researchers also confirmed that the echogenic signals were $\mathrm{pH}$-decreasing dependent by measuring $\mathrm{pH}$ values of the subcutaneous tissues, the SCC-7 tumor xenograft model, and the liver (Fig.3).

In other research, Zhang and coworkers constructed folic acid-conjugated nano-lipid carriers for in situ ultrasound/computed tomography (CT) imaging and tumor photothermal therapy (PTT) [27]. The bubblegenerating nano-lipid carriers were designed by loading gold nanorods, doxorubicin, and ammonium bicarbonate into folic acid-conjugated liposomes. Both in vitro and in vivo experiments revealed that folic acid-conjugated nano-lipid carriers can selectively target more tumor cells 
than doxorubicin and liposomes. Excellent ultrasound contrast enhancement was also observed after nearinfrared (NIR) laser irradiation.

Traditionally, gas cores of the ultrasound contrast agents were used in clinic solely for the purpose of ultrasound imaging. Nowadays, scientists are trying to explore additional functions of gas cores, such as tumor ablation. NBs can pass through tumor blood vessels via the EPR effect, but they may be too small to scatter clinical ultrasonic waves, leading to insufficient ultrasound contrast. Thus, the goal is to develop a nanostructure that can escape from blood vessels to tumor sites, transform intratumorally into MBs for ultrasound imaging, and then collapse to release gas cores to kill tumor cells. Liu et al. developed nanostructures-to-microstructures microbubble bombers for ultrasound imaging and tumor therapy [28]. The nanoplatform consisted of anethole dithiolethione (ADT) (hydrogen sulfide $\left(\mathrm{H}_{2} \mathrm{~S}\right)$ pro-drug) and magnetic nanoliposome (AML), which could be guided by external magnetic field to the interstitial matrix. Spatiotemporally, ADT was catalyzed by cystathionine- $\beta$ synthase (CBS) and cystathionine- $\gamma$-lyase (CSE) in situ to produce $\mathrm{H}_{2} \mathrm{~S}$ bubbles within the tumor microenvironment [29]. When the nanoplatforms were endocytosed by tumor cells, optical microscopy was used to map the realtime $\mathrm{H}_{2} \mathrm{~S}$ bubbles generated by ADT in vitro. After $6 \mathrm{~h}$ of incubation, the cells were disrupted by $\mathrm{H}_{2} \mathrm{~S}$ bubble bombers. In in vivo study, $4 \mathrm{~h}$ after intravenous injection, the researchers found that the echo intensity value of ultrasound with the anethole dithiolethione-loaded group reached almost 3.4-times higher than the blank group. In addition, the nanoplatform showed acceptable safety to normal organs and significantly inhibited tumor growth.

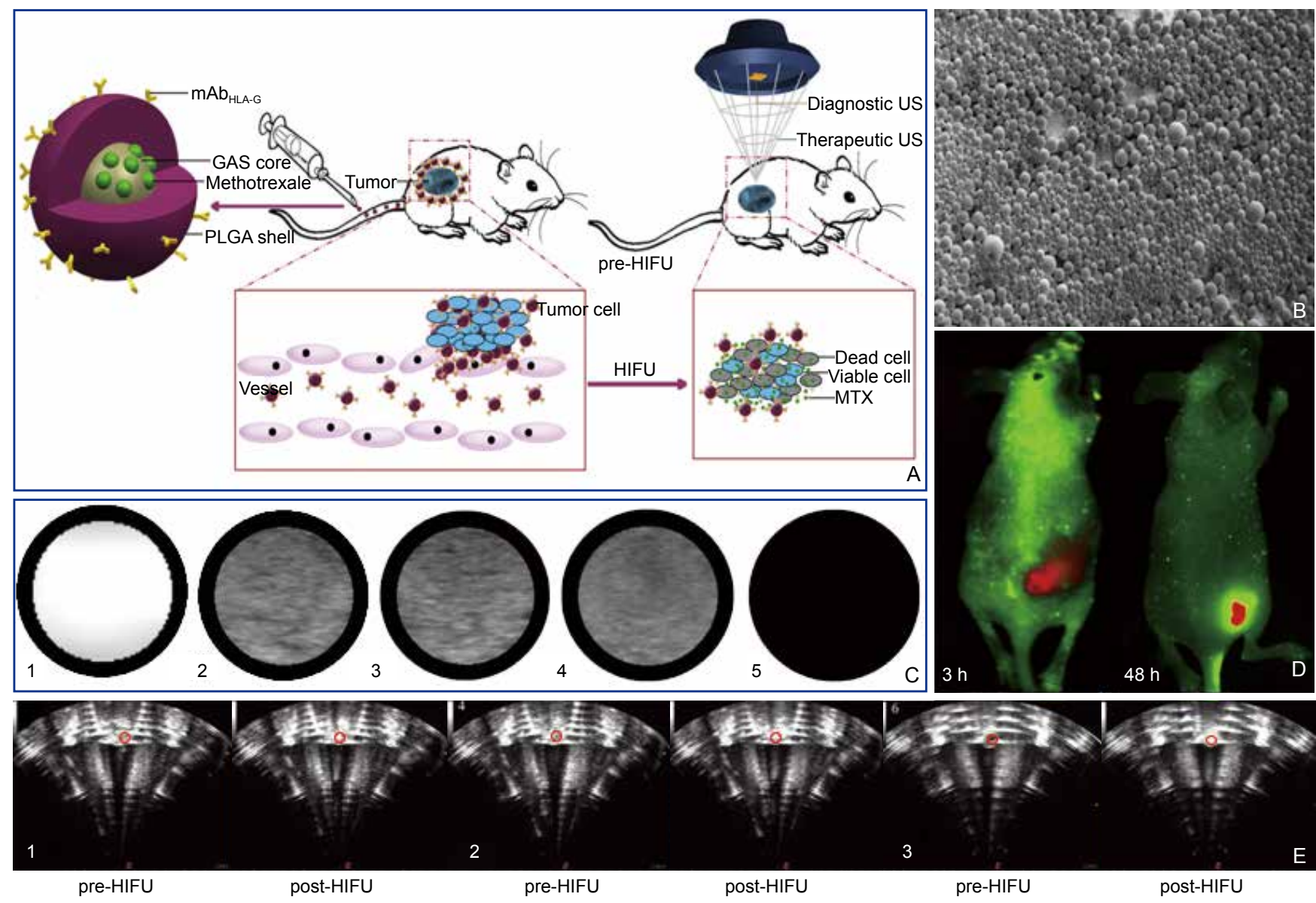

Figure 2 (A) Schematic illustration of the structure of $\mathrm{mAb}_{\text {HLA-G }} /$ MTX/PLGA NBs and how mAb $\mathrm{HLA}_{\mathrm{G}} / \mathrm{MTX} / \mathrm{PLGA}$ NBs act as a synergistic agent improving the treatment efficacy of HIFU ablation for tumor and residual tumor cells; (B) SEM image of mAb HLA-G $_{\text {G }} /$ MTX/PLGA NBs with diameter of $477.6 \pm 119.7 \mathrm{~nm}$; (C) In vitro ultrasound images of the different samples: (1) air (control); (2) $200 \mathrm{mg} / \mathrm{mL} \mathrm{mAb}$ HLA-G $/$ MTX/PLGA NBs; (3) $200 \mathrm{mg} /$ mL pure PLGA NBs; (4) $200 \mathrm{mg} / \mathrm{mL}$ MTX/PLGA NBs; (5) de-gassed and deionized water (control); (D) $0.5 \mathrm{~mL}$ of DiI-labeled mAb $\mathrm{HLA-G}$ /MTX/PLGA NBs are injected via the tail vein, then in vivo imaging of JEG-3 cells in the back of choriocarcinoma subcutaneous transplant sarcoma model by DiIlabeled $\mathrm{mAb}_{\text {HLA-G }} / \mathrm{MTX} / \mathrm{PLGA}$ NBs at $3 \mathrm{~h}$ and $48 \mathrm{~h}$ time point after injection. The results display the target ability of mAb $\mathrm{HLA}_{\text {-G }} / \mathrm{MTX} / \mathrm{PLGA}$ NBs; (E) The diagnostic sensor of HIFU shows the echoes of targeted tissue in the subcutaneous transplant tumor before and after HIFU ablation: (E1) HIFU+saline; (E2) HIFU+MTX/PLGA NBs; (E3) HIFU+mAb HLA-G $_{1} /$ MTX/PLGA NBs. The results indicate that the acoustic signal of tumor tissues in mAb $\mathrm{H}_{\mathrm{HLA}-\mathrm{G}}$-targeted NBs group was significantly higher than those in the saline control group and the non-targeted groups. (Reproduced with permission from [20]) 

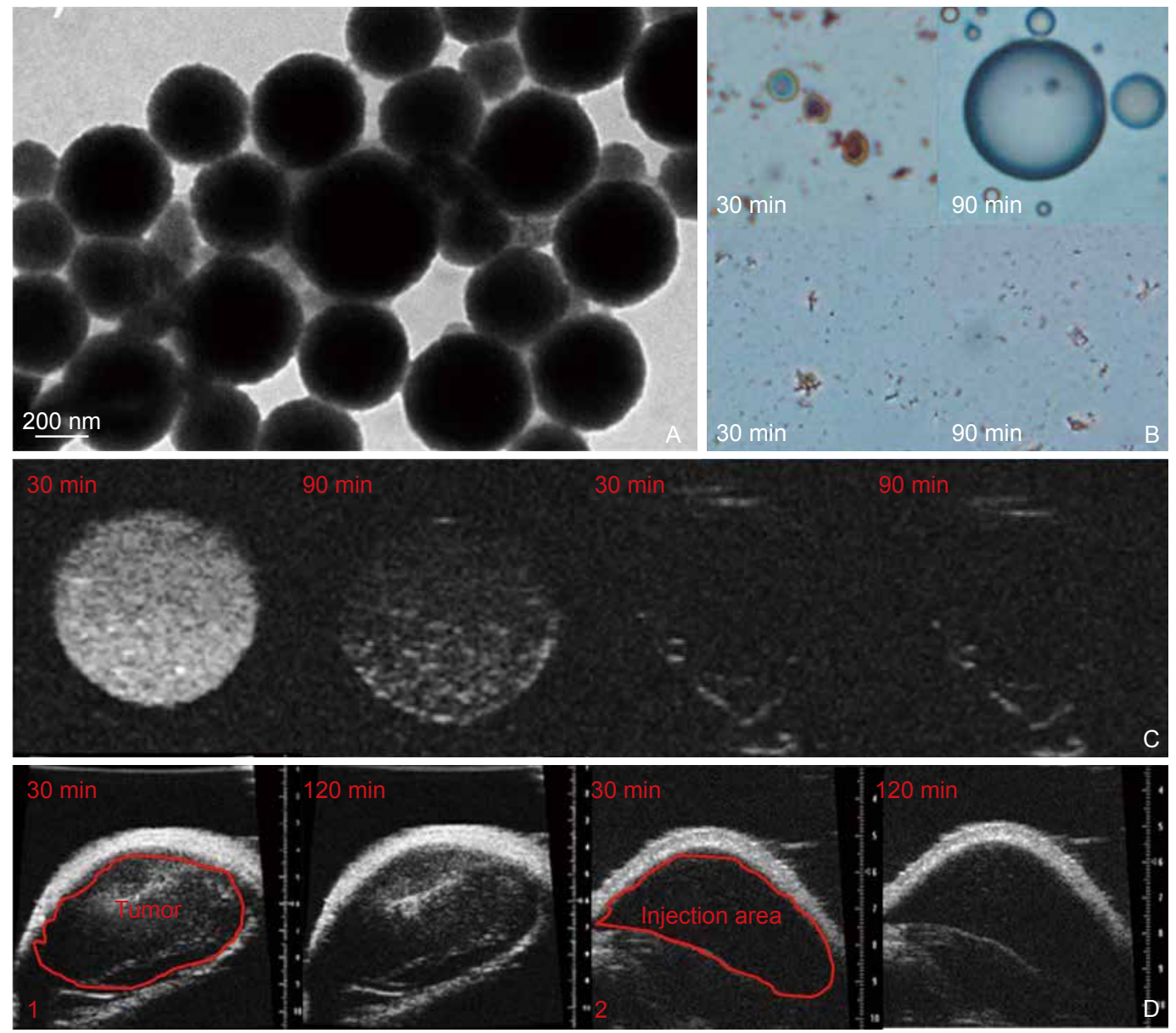

Figure 3 (A) TEM image of DOX-CaCO 3 -MNPs after mineralization for $12 \mathrm{~h}$; (B) Optical micrographs of $\mathrm{CO}_{2}$-generating profiles of sub-micron-size aggregates of $\mathrm{DOX}_{-} \mathrm{CaCO}_{3}-\mathrm{MNPs}$ incubated in $\mathrm{pH}=6.8$ (B1) and $\mathrm{pH}=7.4$ (B2) for 30 and 90 minutes; In vitro ultrasound images of $\mathrm{DOX}^{-\mathrm{CaCO}}{ }_{3}-\mathrm{MNPs}$ incubated in $\mathrm{pH}=6.8(\mathrm{C} 1)$ and $\mathrm{pH}=7.4(\mathrm{C} 2)$ for 30 and 90 minutes; (D1) In vivo ultrasound imaging of the SCC-7 tumor mice by intratumoral injection of DOX-CaCO$-\mathrm{CNNS}_{3}$ for 30 and 120 minutes; (D2) In vivo ultrasound imaging of the SCC-7 tumor-free mice by subcutaneous injection of DOX-CaCO MNPs for 30 and 120 minutes.(Reproduced with permission from [26])

\section{Gene Expression of Ultrasound Contrast Agents in Situ}

Ultrasound also provides a promising method for mapping living organisms within the body. Recently, Shapiro et al. found that gas vesicles genetically encoded by bacteria and archaea can be used as ultrasound contrast [30]. Those gas vesicles, with lengths of $100-600 \mathrm{~nm}$ and width of $45-250 \mathrm{~nm}$, consist of protein shell and gas cores. The gas vesicles show good echogenic ability both in vitro and in vivo. However, the gas vesicles are produced by prokaryotic cells in vitro. And they must be injected through subcutaneous or intravenous to work as ultrasound contrast agents in vivo.

In their another work, Shapiro et.al. imaged the gene expression of tumors in mammalian cells by using the gas vesicles as ultrasound contrast agents [31]. They first screened the gas vesicle-edited genes by synthesizing them from microbial species and then cotransfected them into human embryonic kidney (HEK) 293T cells to identify whether detectable gas vesicles could be expressed or not. After identifying the genes of interest, they linked these genes with the viral cotranslational self-cleavage peptide $\mathrm{P} 2 \mathrm{~A}$ to construct a poly-cistronic mammalian operon. Then, the as-prepared polycistronic operons were incorporated into the cellular genome for consistent gas vesicles expression induced by doxycycline. The expressed gas vesicles with diameters of $64 \pm 12 \mathrm{~nm}$ and lengths of $274 \pm 212 \mathrm{~nm}$ showed characteristic ultrasound contrast ability in vitro. At last, the researchers established the mARG-HEK tumor xenografts in immuno-compromised mice in left flanks and mCherry-HEK control cells in the right flanks. They observed that gas vesicles were expressed by the asprepared polycistronic operons and could be visualized with ultrasound in left flanks after systemic injection of inducer. On the contrary, nothing could be seen from the control side. Interestingly, the gas vesicles could constantly re-express themselves after acoustic collapse. The results demonstrated that the engineered genetic 
construct enabled gene expression imaging in vivo and highlighted the potential for ultrasound to visualize intricate patterns of gene expression noninvasively to monitor cellular location and function inside living organisms (Fig.4).

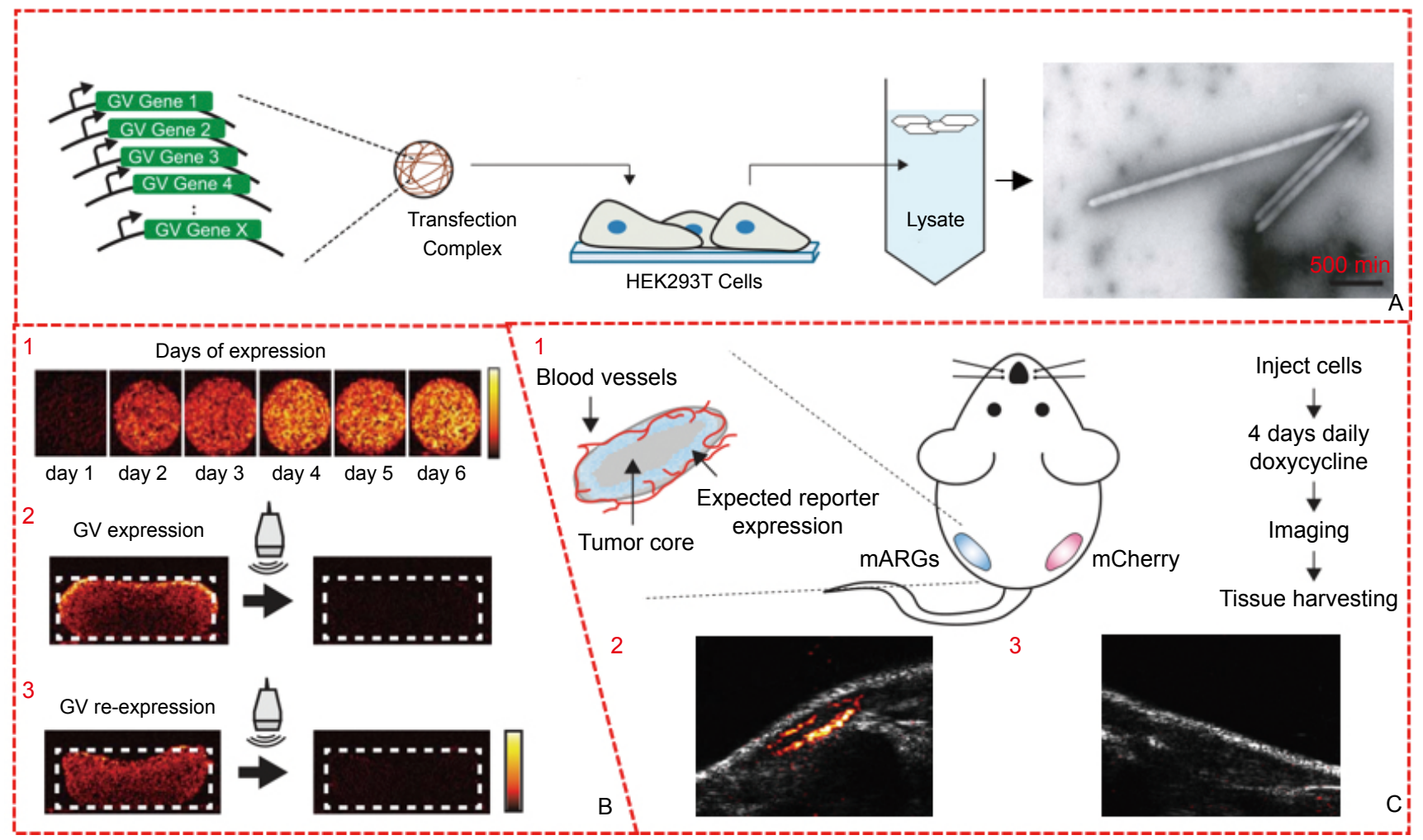

Figure 4 (A) Schematic of the transient cotransfection assay used to identify combinations of genes capable of producing gas vesicles in mammalian cells, and representative TEM image of purified gas vesicles expressed in HEK293Tcells; (B1) Representative ultrasound images and contrast measurements in mARG-HEK cells as a function of time; (B2) Schematic and representative ultrasound images of gas vesicles (GV) expression from mARG-HEK cells in Matrigel; (B3) Re-expression of gas vesicles (GV) after acoustic collapse; (C1) Diagram of a mouse implanted with a subcutaneous tumor model, and the expected spatial pattern of vascularization and doxycycline-induced reporter gene expression; (C2) Representative ultrasound image of tumors containing mARG-HEK cells after 4 days of doxycycline administration. mARG-specific contrast shown in the hot color map is overlaid on an anatomical B-mode image showing the background anatomy; (C3) Representative ultrasound image of tumors containing mCherry-HEK cells after 4 days of doxycycline administration. (Reproduced with permission from [31])

\section{Conclusion}

In this article, we first reviewed the application of NBs acting as ultrasound contrast agents for tumor in situ imaging. Second, we summarized the stimuli-responsive formulations to generate ultrasound contrast agents in situ. Subsequently, we demonstrated the application of gene expression of ultrasound contrast agents in situ to monitor cellular location and function inside living organisms.

In conclusion, in recent decades we have witnessed significant progress in the development of ultrasound contrast agents. It is worthwhile to note that the use of ultrasound contrast agents for imaging tumor in situ has also achieved magnificent outcomes, especially when nanotechnology has been employed. Nevertheless, the following scientific questions currently restrict tumor in situ ultrasound imaging:
(1) The sizes of NBs applied for ultrasound imaging of tumor should be smaller than the size distribution of the tumor vasculature. Creation of small yet highly echogenic NBs still remains to be solved.

(2) There is a need for the ultrasound imaging NBs and nanostructures to be more tissue biocompatible and contain lower toxicity components.

(3) To date, gene expression of gas vessels for ultrasound in situ imaging of tumor is a proof-of-concept method. Efforts should be made to further optimize the genetic constructs so that gene expression of gas vessels for ultrasound in situ imaging are more sensitive and practical.

\section{Acknowledgments}

This work was supported by the National Key Research and Development Program of China (2016YFA0201400), National Natural 
Science Foundation of China (81822022, 81771846, 81571810), and Beijing Talents Foundation (2018000021223ZK48), and Grants from Peking University Third Hospital (BYSYZD2019018, jyzc2018-02, and BYSY2015023).

\section{Conflict of Interest}

There is no conflict of interest and no disclosure associated with the manuscript.

\section{References:}

[1] Gramiak R. Echocardiography of the aortic root. Investigative Radiology. 1968; 3:356-66.

[2] Sponheim N, Hoff L, A. Waaler A, Muan B, Morris H, Holm S. Albunex-a new ultrasound contrast agent. International Conference on Acoustic Sensing \& Imaging 1993; 1:103-8.

[3] Li G, Wang Q, Zhao X. Introduction of ultrasound microbubble technology aided tumor imaging and treatment. IEEE International Conference on Medical Imaging Physics and Engineering 2014; 4:192-5.

[4] Perera RH, Hernandez C, Zhou H, Kota P, Burke A, Exner AA. Ultrasound imaging beyond the vasculature with new generation contrast agents. Wiley Interdisciplinary Reviews Nanomedicine \& Nanobiotechnology 2015; 7:593-608.

[5] Ferrara KW, Borden MA, Zhang H. Lipid-shelled vehicles: engineering for ultrasound molecular imaging and drug delivery. Accounts of Chemical Research 2009; 42:881-892.

[6] Sheeran PS, Luois S, Matsunaga TO. Submicron decafluorobutane phase-change contrast agents generated by microbubble condensation. Ultrasonics Symposium (IUS) 2011; pp:636-9.

[7] Jian J, Liu C, Gong Y, Su L, Zhang B, Wang Z. India ink incorporated multifunctional phase-transition nanodroplets for photoacoustic/ ultrasound dual-modality imaging and photoacoustic effect based tumor therapy. Theranostics 2014; 4:1026-1038.

[8] Liu J, Zhang B, Li M, Zhou M, Li F, Huang X, et al. Preparation and characterization of a novel silicon-modified nanobubble. Plos one 2017; 12:31-44.

[9] Yu H, Wang W, He X, Zhou Q, Ding M. Novel fluorescence nanobubbles for contrast-enhanced ultrasound imaging in rabbit VX2 hepatocellular carcinoma model. International Society for Optics and Photonics 2017; 10139:1-10.

[10] Jin Q, Lin CY, Kang ST, Chang YC, Zheng H, Yang CM, et al, Superhydrophobic silica nanoparticles as ultrasound contrast agents. Ultrasonics Sonochemistry 2017; 36:262-269.

[11] Lakshmanan A, Lu GJ, Farhadi A, Nety SP, Kunth M, Lee-Gosselin A, et al. Preparation of biogenic gas vesicle nanostructures for use as contrast agents for ultrasound and MRI. Nature Protocols 2017; 12:2050-2080.

[12] Shapiro MG. Genetically encoded gas nanostructures as biophyically tunable molecular reporters for mri and ultrasound. Biophysical Journal 2014; 106:19.

[13] Hobbs SK, Monsky WL, Yuan F, Roberts WG, Griffith L, Torchilin VP. Regulation of transport pathways in tumor vessels: role of tumor type and microenvironment. Biomaterials 2012; 33:5854-5864.

[14] Zhou Y, Wang Z, Chen Y, Shen H, Luo Z, Li A, et al. Microbubbles from gas-generating perfluorohexane nanoemulsions for targeted temperature-sensitive ultrasonography and synergistic HIFU ablation of tumors.' Advanced Materials 2013; 25:4123-30.

[15] Guo R, Tian Y, Wang Y, Yang W. Near - infrared laser - triggered nitric oxide nanogenerators for the reversal of multidrug resistance in cancer. Advanced Functional Materials 2017; 27:160398-404.

[16] Hobbs SK, Monsky WL, Yuan F, Roberts WG, Griffith L, Torchilin VP. Regulation of transport pathways in tumor vessels: Role of tumor type and microenvironment. Proceedings of the National Academy of Sciences of the United States of America 1998; 95:4607-12.

[17] Li C, Wu K, Jing L, Liu H, Zhou Q, Ding M, et al. A preliminary evaluation of self-made nanobubble in contrast-enhanced ultrasound imaging. The International Society for Optical Engineering 2014; 9038:11-18.

[18] Zheng R, Yin T, Wang P, Zheng B, Cheng D. Nanobubbles for enhanced ultrasound imaging of tumor. Int J Nanomedicine 2012; 7:895-904.

[19] Yang H, Cai W, Xu L, Lv X, Qiao Y, Li P, et al. NanobubbleAffibody: Novel ultrasound contrast agents for targeted molecular ultrasound imaging of tumor. Biomaterials 2014; 37:279-88.

[20] Zhang X, Zheng Y, Wang Z, Huang S, Chen Y, Jiang W, et al. Methotrexate-loaded PLGA nanobubbles for ultrasound imaging and Synergistic Targeted therapy of residual tumor during HIFU ablation. Biomaterials 2014; 35:5148-61.

[21] Bloch SH, Wan M, Dayton PA. Optical observation of lipid- and polymer-shelled ultrasound microbubble contrast agents. Applied Physics Letters 2004; 84:631-33.

[22] Min HS, Kang E, Koo H, Lee J, Kim K, Park RW, et al. Gasgenerating polymeric microspheres for long-term and continuous in vivo ultrasound imaging. Biomaterials 2012; 33:936-44.

[23] Nieves L, Hernandez C, Nittayacharn P, Lilly J, Coyne R, et al. Effect of the surfactant pluronic on the stability of lipid-stabilized perfluorocarbon nanobubbles. International Ultrasonics Symposium (IUS) 2017; pp: 1-4

[24] Browning RJ, Bian S, Reardon PJ. Ultrasound enhanced delivery of cisplatin loaded nanoparticles. Journal of the Acoustical Society of America 2017, 141:3459-60.

[25] Sloun RJGV, Solomon O, Eldar YC. Sparsity-driven super-resolution in clinical contrast-enhanced ultrasound. International Ultrasonics Symposium (IUS) 2017; pp: 236-9.

[26] Min KH, Min HS, Lee HJ, Park DJ, Yhee JY, Kim K, et al. pHcontrolled gas-generating mineralized nanoparticles: a theranostic agent for ultrasound imaging and therapy of Cancers. Acs Nano 2015; 9:134-45.

[27] Zhang N, Li J, Hou R, Zhang J, Wang P, Liu X, et al. Bubblegenerating nano-lipid carriers for ultrasound/CT imaging-guided efficient tumor therapy. Int J Pharm 2017; 20:251-62.

[28] Liu Y, Yang F, Yuan C, Li M, Wang T, Chen B, et al. Magnetic nanoliposomes as in situ microbubble bombers for multimodality image-guided cancer theranostics. Acs Nano 2017; 11:1509-19.

[29] Kashfi K, Olson KR. Biology and therapeutic potential of hydrogen sulfide and hydrogen sulfide-releasing chimeras. Biochemical Pharmacology 2013; 85:689-703.

[30] Mikhail G. Shapiro, Patrick W. he, Arkosnato Neogy, Melissa Yin, F. Stuart Foster, David V. Schaffer. Biogenic gas nanostructures as ultrasonic molecular reporters. Nature Nanotechnology 2014; 9:311316.

[31] Farhadi A, Ho GH, Sawyer DP, Bourdeau RW, Shapiro MG. Ultrasound imaging of gene expression in mammalian cells. Science $2019 ; 365: 1469-75$. 